\title{
An Approximation Approach to Eigenvalue Intervals for Singular Boundary Value Problems with Sign Changing and Superlinear Nonlinearities
}

\author{
Haishen Lü, ${ }^{1}$ Ravi P. Agarwal, ${ }^{2,3}$ and Donal O'Regan' \\ ${ }^{1}$ Department of Applied Mathematics, Hohai University, Nanjing 210098, China \\ ${ }^{2}$ Department of Mathematical Sciences, Florida Institute of Technology, Melbourne, FL 32901-6975, USA \\ ${ }^{3}$ KFUPM Chair Professor, Mathematics and Statistics Department, King Fahd University of Petroleum and \\ Minerals, Dhahran 31261, Saudi Arabia \\ ${ }^{4}$ Department of Mathematics, National University of Ireland, Galway, Ireland
}

Correspondence should be addressed to Haishen Lü, haishen2001@yahoo.com.cn

Received 25 June 2009; Accepted 5 October 2009

Recommended by Ivan T. Kiguradze

This paper studies the eigenvalue interval for the singular boundary value problem $-u^{\prime \prime}=g(t, u)+$ $\lambda h(t, u), t \in(0,1), u(0)=0=u(1)$, where $g+h$ may be singular at $u=0, t=0,1$, and may change sign and be superlinear at $u=+\infty$. The approach is based on an approximation method together with the theory of upper and lower solutions.

Copyright (C) 2009 Haishen Lü et al. This is an open access article distributed under the Creative Commons Attribution License, which permits unrestricted use, distribution, and reproduction in any medium, provided the original work is properly cited.

\section{Introduction}

The singular boundary value problems of the form

$$
\begin{gathered}
-u^{\prime \prime}=f(t, u), \quad t \in(0,1) \\
u(0)=0=u(1)
\end{gathered}
$$

occurs in several problems in applied mathematics, see [1-6] and their references. In many papers, a critical condition is that

$$
f(t, r) \geq 0 \quad \text { for }(t, r) \in(0,1) \times(0, \infty)
$$


or there exists a constant $L>0$ such that for any compact set $K \subset(0,1)$, there is $\varepsilon=\varepsilon_{K}>0$ such that

$$
\begin{gathered}
f(t, r) \geq L \quad \forall t \in K, r \in(0, \varepsilon], \\
\lim _{r \rightarrow \infty} \frac{f(t, r)}{r}=0 \quad \forall t \in(0,1) .
\end{gathered}
$$

We refer the reader to [1-4]. In the case, when $f(t, r)$ may change sign in a neighborhood of $r=0$ and $\lim \sup _{r \rightarrow+\infty}(f(t, r) / r)=+\infty$ for $t \in(0,1)$, very few existence results are available in literature [1].

In this paper we study positive solutions of the second boundary value problem

$$
\begin{gathered}
-u^{\prime \prime}=g(t, u)+\lambda h(t, u), \quad t \in(0,1), \\
u(0)=0=u(1) ;
\end{gathered}
$$

here $g:(0,1) \times(0, \infty) \rightarrow R$ and $h:(0,1) \times[0, \infty) \rightarrow(0, \infty)$ are continuous, so as a result, our nonlinearity may be singular at $t=0,1$ and $u=0$. Also our nonlinearity may change sign and be superlinear at $u=+\infty$. Our main existence results (Theorems 1.1, 1.2 and 1.4) are new (see Remark 1.5, Examples 3.1 and 3.2).

A function $u$ is a solution of the boundary value problem (1.4) if $u:[0,1] \rightarrow R, u$ satisfies the differential equation $(1.4)$ on $(0,1)$ and the stated boundary data.

Let $C[0,1]$ denote the class of maps $u$ continuous on $[0,1]$, with norm $|u|_{\infty}=$ $\max _{t \in[0,1]}|u(t)|$. We put $\min \{a, b\}=a \wedge b ; \max \{a, b\}=a \vee b$. Given $\alpha, \beta \in C[0,1], \alpha \leq \beta$, let

$$
D_{\alpha}^{\beta}=\{v \mid v \in C[0,1], \alpha \leq v \leq \beta\}
$$

Let

$$
M=\left\{h \in C(0,1): \int_{0}^{1}|h(s)| d s<\infty \text { with } \lim _{t \rightarrow 0^{+}}|h(t)|<\infty, \lim _{t \rightarrow 1^{-}}(1-t)|h(t)|<\infty\right\} .
$$

In this paper, we suppose the following conditions hold:

(G1) suppose there exist $g_{i}:(0,1) \times(0, \infty) \rightarrow(0, \infty)(i=1,2)$ continuous functions such that

$$
\begin{gathered}
g_{i}(t, \cdot) \text { is strictly decreasing for } t \in(0,1), \\
g_{1}\left(\cdot, r \phi_{1}(\cdot)\right), \quad g_{2}(\cdot, r) \in M \quad \forall r>0, \\
-g_{1}(t, r) \leq g(t, r) \leq g_{2}(t, r) \quad \text { for }(t, r) \in(0,1) \times(0, \infty),
\end{gathered}
$$

where $\phi_{1}$ is defined in Lemma 2.1; 
$(H 1)$ there exist $h_{i}:(0,1) \times[0, \infty) \rightarrow[0, \infty)(i=1,2)$ continuous functions such that

$$
\begin{gathered}
h_{i}(t, \cdot) \text { is increasing for } t \in(0,1), \\
h_{1}(\cdot, r), h_{2}(\cdot, r) \in M \text { for } r>0, \\
h_{1}(t, r) \leq h(t, r) \leq h_{2}(t, r) \text { for }(t, r) \in(0,1) \times[0, \infty) ;
\end{gathered}
$$

(H2) there exists $\bar{r}>0$ such that $h_{1}(t, \bar{r})>0$ for $t \in(0,1)$.

The main results of the paper are the following.

Theorem 1.1. Suppose (G1), (H1), (H2) and the following conditions hold:

(G2) for all $r_{2}>r_{1}>0$, there exists $\gamma(\cdot) \in M$ such that $g_{2}(\cdot, r)+\gamma(\cdot) r$ is increasing in $\left(r_{1}, r_{2}\right)$ :

$$
\lim _{r \rightarrow \infty} \frac{h_{1}(t, r)}{r}=0 \quad \forall t \in(0,1)
$$

(H4) there exists a sequence $\left\{R_{j}\right\}_{j=1}^{\infty}$ such that $\lim _{j \rightarrow \infty} R_{j}=\infty$ and

$$
\lim _{j \rightarrow \infty} \frac{h_{2}\left(s, R_{j}+a_{1}\right)}{R_{j}}=0,
$$

where $a_{1}=1+\int_{0}^{1} g_{2}(s, 1) d s$.

Then there exists $\lambda_{1}^{*}>0$ such that for every $\lambda \geq \lambda_{1}^{*}$, (1.4) has at least one positive solution $u \in C[0,1] \cap C^{1}(0,1)$ and $u>0$ for $t \in(0,1)$.

Theorem 1.2. Suppose (G1), (H1), (H2) and the following conditions hold:

(G3) for all $r_{2}>r_{1}>0$ there exists $\gamma(\cdot) \in M$ such that $g(t, r)+\gamma(t) r$ is increasing in $\left(r_{1}, r_{2}\right)$;

(G4) there exists $c_{1}>0$ such that

$$
0 \leq g(t, r), \quad t \in(0,1), 0<r<c_{1}
$$

(G5) there exists $c_{2} \in\left(0, c_{1}\right), 0<\beta<1$ such that for all $r \in\left(0, c_{2}\right)$

$$
\int_{0}^{1} t(1-t) \bar{g}_{1}(t, r l(t)) d t \geq r \pi
$$

where

$$
\bar{g}_{m}(t, r)=\min \left\{g(t, r), \frac{m}{r^{\beta}}\right\} \text { for } m \geq 1,
$$

and $l(t)=\min \{t, 1-t\}$ for $t \in[0,1]$. 
Then there exists $\lambda_{2}^{*}>0$ such that

(i) if $0<\lambda<\lambda_{2}^{*}$, (1.4) has at least one solution $u \in C[0,1] \cap C^{1}(0,1)$ and $u>0$ for $t \in(0,1)$;

(ii) if $\lambda>\lambda_{2}^{*}$, (1.4) has no solutions.

Remark 1.3. Notice that $\bar{g}_{m}(t, r)$ satisfies (G1), (G3), (G4) and for fixed $m \geq 1$,

$$
\begin{gathered}
\int_{0}^{1} t(1-t) \bar{g}_{m}(t, r l(t)) d t \geq r \pi \quad \text { for } r \in\left(0, c_{2}\right), \\
g(t, r) \geq \bar{g}_{m}(t, r) \geq \bar{g}_{1}(t, r) \text { for } t \in(0,1), r \in(0, \infty) .
\end{gathered}
$$

Theorem 1.4. Suppose (G1), (H1), (H2) and the following conditions hold:

(G6) there exists $\tau \geq \tau_{1}$ such that

$$
\lim _{r \rightarrow 0^{+}} \frac{\tau r+g^{-}(t, r)}{h(t, r)}=0
$$

where $\tau_{1}$ is defined in Lemma 2.1 and $g^{+}(t, r)=\max \{0, g(t, r)\}, g^{-}(t, r)=\max \{0$, $-g(t, r)\}$;

(H5) for all $r_{2}>r_{1}>0$, there exists $\gamma(\cdot) \in M$ such that $h(t, r)+\gamma(t) r$ is increasing in $\left(r_{1}, r_{2}\right)$.

Then there exists $\lambda_{3}^{*}>0$ such that

(i) if $0<\lambda<\lambda_{3}^{*}$, (1.4) has at least one solution $u \in C[0,1] \cap C^{1}(0,1)$ and $u>0$ for $t \in(0,1)$;

(ii) if $\lambda>\lambda_{3}^{*}$, (1.4) has no solutions.

Remark 1.5. In $[5,6]$, the authors consider the boundary value problem (1.4) under the conditions

$$
\lim _{r \rightarrow \infty} \frac{h_{2}(t, r)}{r}=0
$$

In Section 3, we give two examples (see Examples 3.1 and 3.2) which satisfy the conditions in Theorem 1.1 or Theorem 1.2 but they do not satisfy the conditions in [1-5].

\section{Proof of Main Results}

\subsection{Some Lemmas}

Lemma 2.1. Consider the following eigenvalue problem

$$
\begin{gathered}
-u^{\prime \prime}=\tau u(t), \quad t \in(0,1), \\
u(0)=u(1)=0 .
\end{gathered}
$$


Then the eigenvalues are

$$
\tau_{m}=(m \pi)^{2} \quad \text { for } m=1,2, \ldots
$$

and the corresponding eigenfunctions are

$$
\phi_{m}(t)=\sin m \pi t \quad \text { for } m=1,2, \ldots
$$

Let $G(t, s)$ be the Green's function for the BVP:

$$
\begin{gathered}
-u^{\prime \prime}=0 \quad \text { for } t \in(0,1) \\
u(0)=u(1)=0 .
\end{gathered}
$$

Then

$$
G(t, s)= \begin{cases}s(1-t), & 0 \leq s<t \leq 1 \\ t(1-s), & 0 \leq t<s \leq 1\end{cases}
$$

Also for all $(t, s) \in[0,1] \times[0,1]$, define

$$
N(t, s)= \begin{cases}\frac{G(t, s)}{\phi_{1}(t)} & \text { if } t \neq 0,1 \\ \frac{1-s}{\pi} & \text { if } t=0 \\ \frac{s}{\pi} & \text { if } t=1\end{cases}
$$

It follows easily that

$$
\begin{aligned}
& 0<G(t, s) \leq t(1-t) \quad \text { for }(t, s) \in(0,1) \times(0,1) \\
& \frac{s(1-s)}{2 \pi} \leq N(t, s) \leq \frac{1}{2} \quad \text { for }(t, s) \in(0,1) \times(0,1)
\end{aligned}
$$

Define the operator $A, B: M \rightarrow C[0,1]$ by

$$
\begin{aligned}
& A x(t)=\int_{0}^{1} G(t, s) x(s) d s \\
& B x(t)=\int_{0}^{1} N(t, s) x(s) d s .
\end{aligned}
$$

The following four results can be found in [5] (notice $\lim _{r \rightarrow \infty}\left(h_{2}(t, r) / r\right)=0$ is not needed in the proofs there). 
Lemma 2.2. Suppose (G1) and (H1) hold. Let $n_{0} \in N$. Assume that for every $n>n_{0}$, there exist $a_{n}, \delta_{n}, \delta \in M$ such that

$$
0 \leq a_{n}(t), \quad\left|\delta_{n}(t)\right| \leq \delta(t), \quad \lim _{n \rightarrow \infty} \delta_{n}(t)=0, \quad \text { for } t \in(0,1)
$$

and there exist $\bar{u}, \bar{u}_{n}, \widehat{u}_{n}, \widehat{u} \in C[0,1]$ such that

$$
0<\bar{u}(t) \leq \bar{u}_{n}(t) \leq \widehat{u}_{n}(t) \leq \widehat{u}(t) \quad \text { for } t \in(0,1)
$$

and $\widehat{u}(0)=\widehat{u}(1)=0$. If

$$
\begin{aligned}
& -\bar{u}_{n}^{\prime \prime}(t)+a_{n}(t) \bar{u}_{n}(t) \\
& \quad \leq g\left(t, \frac{1}{n}+v\right)+\lambda h(t, v)+\delta_{n}(t)+a_{n}(t) v(t) \quad \text { for } t \in(0,1), \\
& -\widehat{u}_{n}^{\prime \prime}(t)+a_{n}(t) \widehat{u}_{n}(t) \\
& \quad \geq g\left(t, \frac{1}{n}+v\right)+\lambda h(t, v)+\delta_{n}(t)+a_{n}(t) v(t) \quad \text { for } t \in(0,1),
\end{aligned}
$$

where $\lambda \geq 0$ and $v \in D_{\bar{u}_{n}}^{\widehat{u}_{n}}$, then (1.4) has a solution $u \in C[0,1] \cap C^{1}(0,1)$ such that $\bar{u}(t) \leq u(t) \leq \widehat{u}(t)$ for $t \in[0,1]$.

Lemma 2.3. Let $\psi:(0,1) \times(0, \infty) \rightarrow(0, \infty)$ be a continuous function with

$$
\begin{gathered}
\psi(t, \cdot) \text { is strictly decreasing, } \\
\psi(\cdot, r) \in M \quad \forall r>0 .
\end{gathered}
$$

Then the problem

$$
\begin{gathered}
-\omega^{\prime \prime}(t)=\psi\left(t, \omega(t)+\frac{1}{n}\right) \quad \text { for } t \in(0,1) \\
\omega(0)=\omega(1)=0
\end{gathered}
$$

has a solution $\omega_{n} \in C[0,1]$ such that

$$
\omega_{n}(t) \leq \omega_{n+1}(t) \leq 1+\omega_{1}(t) \leq 1+\int_{0}^{1} \psi(s, 1) d s \quad \text { for } t \in[0,1], n \in N .
$$

If we let $\omega(t)=\lim _{n \rightarrow \infty} \omega_{n}(t)$ for $t \in[0,1]$, then

$$
\begin{gathered}
\omega \in C[0,1], \quad \omega(t)>0 \quad \text { for } t \in(0,1), \\
-\omega^{\prime \prime}(t)=\psi(t, \omega(t)) \quad \text { for } t \in(0,1), \\
\omega(0)=\omega(1)=0 .
\end{gathered}
$$


Next we consider the boundary value problem

$$
\begin{gathered}
-u^{\prime \prime}+a(t) u(t)=f(t), \quad t \in(0,1), \\
u(0)=0=u(1)
\end{gathered}
$$

where $a, f \in M, a(t) \geq 0$ for $t \in(0,1)$.

Lemma 2.4. The following statements hold:

(i) for any $f \in M,(2.16)$ is uniquely solvable and

$$
u+A(a u)=A(f)
$$

(ii) if $f(t) \geq 0$ for $t \in(0,1)$, then the solution of (2.16) is nonnegative.

Corollary 2.5. Let $\Phi: M \rightarrow C[0,1] \cap C^{1}(0,1)$ be the operator such that $\Phi(f)$ is the solution of (2.16). Then we have

(i) if $f_{1}(t) \leq f_{2}(t)$ for $t \in(0,1)$, then $\Phi\left(f_{1}\right)(t) \leq \Phi\left(f_{2}\right)(t)$ for $t \in[0,1]$;

(ii) let $E \subset M$ and $\beta \in M$. If $|f(t)| \leq \beta(t), t \in(0,1)$ for all $f \in E$, then $\Phi(E)$ is relatively compact with respect to the topology of $C[0,1]$.

Lemma 2.6 (see [2]). Let $f \in M, f \geq 0, f \not \equiv 0, u \in C[0,1] \cap C^{1}(0,1)$ satisfy

$$
\begin{gathered}
-u^{\prime \prime}=f \quad \text { in }(0,1), \\
u(0)=u(1)=0 .
\end{gathered}
$$

Then there exist $m=m(f)>0, M=M(f)>0$ such that

$$
m l(t) \leq u(t) \leq M l(t) \quad \text { for } t \in[0,1]
$$

\subsection{The Proof of Theorem 1.1}

Claim 1 (see [5]). There exists $\lambda_{1}^{*}>0, c>0$, independent of $\lambda$, such that for all $\lambda \geq \lambda_{1}^{*}$ there exist $R_{\curlywedge}>c, \bar{u} \in C([0,1])$, with $c \phi_{1}(t) \leq \bar{u}(t) \leq R_{\curlywedge} \phi_{1}(t)$ and

$$
\begin{gathered}
-\bar{u}^{\prime \prime}(t)=-g_{1}(t, \bar{u}(t))+\lambda h_{1}(t, \bar{u}(t)), \quad \text { for } t \in(0,1), \\
\bar{u}(0)=\bar{u}(1)=0,
\end{gathered}
$$


with

$$
g_{1}(\cdot, \bar{u}(\cdot)), h_{1}(\cdot, \bar{u}(\cdot)) \in M
$$

Let $\lambda_{1}^{*}>0, c>0$ and $\bar{u} \in C[0,1]$ be defined in Claim 1. Define

$$
\psi(t, r)=g_{2}(t, r) \quad \text { for } t \in(0,1)
$$

From (G1) notice that $\psi$ satisfies the assumptions of Lemma 2.3, so there exist $\omega, \omega_{n} \in$ $C[0,1], \omega_{n}(t)>0, \omega(t)>0$ for $t \in(0,1)$ such that

$$
\begin{gathered}
-\omega_{n}^{\prime \prime}(t)=g_{2}\left(t, \frac{1}{n}+\omega_{n}\right) \quad \text { for } t \in(0,1), \\
\omega_{n}(0)=\omega_{n}(1)=0, \\
\omega_{n}(t) \leq \omega_{n+1}(t) \leq 1+\omega_{1}(t) \leq a_{1} \quad \text { for } t \in[0,1], n \in N, \\
\omega(t)=\lim _{n \rightarrow \infty} \omega_{n}(t) \quad \text { for } t \in[0,1], \\
-\omega^{\prime \prime}(t)=g_{2}(t, \omega(t)) \quad \text { for } t \in(0,1), \\
\\
\omega(0)=\omega(1)=0,
\end{gathered}
$$

where $a_{1}=1+\int_{0}^{1} g_{2}(s, 1) d s$.

Let $\lambda \geq \lambda_{1}^{*}, n \in N$ be fixed. We consider the following boundary value problem:

$$
\begin{gathered}
-v^{\prime \prime}(t)=\lambda h_{2}\left(t, v+\omega_{n}\right)+\lambda h_{1}(t, \bar{u}) \quad \text { for } t \in(0,1), \\
v(0)=v(1)=0 .
\end{gathered}
$$

By $(H 4)$, there exist $\left\{R_{j}\right\}_{j=1}^{\infty}$ such that $\lim _{j \rightarrow \infty} R_{j}=\infty$ and

$$
\lim _{j \rightarrow \infty} \frac{h_{2}\left(t, R_{j}+a_{1}\right)}{R_{j}}=0 \quad \text { for } t \in(0,1)
$$

so

$$
\lim _{j \rightarrow \infty} \frac{\lambda h_{2}\left(t, R_{j}+a_{1}\right)+\lambda h_{1}(t, \bar{u}(t))}{R_{j}}=0 \quad \text { for } t \in(0,1) .
$$


There exists $j_{0} \in N$ such that

$$
\lambda h_{2}\left(t, R_{j_{0}}+a_{1}\right)+\lambda h_{1}(t, \bar{u}(t)) \leq R_{j_{0}}
$$

If $v \in C[0,1]$ and $0 \leq v(t) \leq R_{j_{0}} \phi_{1}(t)$ for $t \in[0,1]$, then

$$
\begin{aligned}
& \int_{0}^{1} N(t, s)\left[\lambda h_{2}\left(s, v(s)+\omega_{n}(s)\right)+\lambda h_{1}(s, \bar{u})\right] d s \\
& \quad \leq \int_{0}^{1} N(t, s)\left[\lambda h_{2}\left(s, v(s)+a_{1}\right)+\lambda h_{1}(s, \bar{u})\right] d s \\
& \quad \leq \int_{0}^{1} N(t, s)\left[\lambda h_{2}\left(s, R_{j_{0}} \phi_{1}(s)+a_{1}\right)+\lambda h_{1}(s, \bar{u})\right] d s \\
& \quad \leq \int_{0}^{1} N(t, s)\left[\lambda h_{2}\left(s, R_{j_{0}}+a_{1}\right)+\lambda h_{1}(s, \bar{u})\right] d s \\
& \quad \leq \frac{R_{j_{0}}}{2}, \quad \text { for } t \in(0,1),
\end{aligned}
$$

and so

$$
0 \leq \int_{0}^{1} G(t, s)\left[\lambda h_{2}\left(s, v(s)+\omega_{n}(s)\right)+\lambda h_{1}(s, \bar{u}(s))\right] d s \leq R_{j_{0}} \phi_{1}(t) \quad \text { for } t \in[0,1]
$$

Let $\Phi: C[0,1] \rightarrow C[0,1]$ be the operator defined by

$$
(\Phi v)(t):=\int_{0}^{1} G(t, s)\left[\lambda h_{2}\left(s, v(s)+\omega_{n}(s)\right)+\lambda h_{1}(s, \bar{u}(s))\right] d s \quad \text { for } v \in C[0,1], t \in[0,1]
$$

It is easy to see that $\Phi$ is a continuous and completely continuous operator. Also if $0 \leq v(t) \leq$ $R_{j_{0}} \phi_{1}(t)$ for $t \in[0,1]$, then $0 \leq \Phi(v)(t) \leq R_{j_{0}} \phi_{1}(t)$ for $t \in[0,1]$, so Schauder's fixed point theorem guarantees that there exists $\widetilde{v} \in\left[0, R_{j_{0}} \phi_{1}\right]$ such that $\Phi(\widetilde{v})=\widetilde{v}$, that is,

$$
\begin{gathered}
-\widetilde{v}^{\prime \prime}(t)=\lambda h_{2}\left(t, \widetilde{v}(t)+\omega_{n}(s)\right)+\lambda h_{1}(t, \bar{u}(t)), \\
\widetilde{v}(1)=\widetilde{v}(1)=0 .
\end{gathered}
$$

Let

$$
\widehat{u}_{n}(t)=\omega_{n}(t)+\widetilde{v}_{n}(t) \quad \text { for } t \in[0,1]
$$


Then $\widehat{u}_{n} \in C[0,1], \widehat{u}_{n}(1)=\widehat{u}_{n}(1)=0$, and

$$
\begin{aligned}
-\widehat{u}_{n}^{\prime \prime}(t) & =-\omega_{n}^{\prime \prime}(t)-\widetilde{v}_{n}^{\prime \prime}(t) \\
& =g_{2}\left(t, \frac{1}{n}+\omega_{n}\right)+\lambda h_{2}\left(t, \omega_{n}+\widetilde{v}_{n}\right)+\lambda h_{1}(t, \bar{u}) \\
& \geq g_{2}\left(t, \frac{1}{n}+\widehat{u}_{n}\right)+\lambda h_{1}(t, \bar{u})+\lambda h_{2}\left(t, \widehat{u}_{n}\right) \quad \text { for } t \in(0,1) .
\end{aligned}
$$

Let

$$
\widehat{u}(t)=\omega(t)+R_{j_{0}} \phi_{1}(t) \quad \text { for } t \in[0,1],
$$

so

$$
0 \leq \widehat{u}_{n}(t) \leq \widehat{u}(t) \quad \text { for } t \in[0,1] .
$$

From Claim 1, we obtain

$$
\begin{aligned}
-\bar{u}^{\prime \prime}(t) & =-g_{1}(t, \bar{u})+\lambda h_{1}(t, \bar{u}) \\
& \leq \lambda h_{1}(t, \bar{u}) \\
& \leq \lambda h_{1}(t, \bar{u})+g_{2}\left(t, \frac{1}{n}+\widehat{u}_{n}\right)+\lambda h_{2}\left(t, \widehat{u}_{n}\right) \\
& \leq-\widehat{u}_{n}^{\prime \prime}(t) \quad \text { for } t \in(0,1),
\end{aligned}
$$

that is,

$$
-\left(\bar{u}-\widehat{u}_{n}\right)^{\prime \prime}(t) \leq 0 \quad \text { for } t \in(0,1) .
$$

A standard argument yields

$$
\bar{u}(t) \leq \widehat{u}_{n}(t) \quad \text { for } t \in[0,1] .
$$

From (G2), there exists $\gamma \in M$ such that $r \rightarrow g_{2}(t, 1 / n+r)+\gamma(t) r$ is increasing on $\left(0,|\widehat{u}|_{\infty}\right)$. Let $\bar{u}_{n}=\bar{u}$. From (2.35) and (2.38), we have

$$
0<\bar{u}(t) \leq \bar{u}_{n}(t) \leq \widehat{u}_{n}(t) \leq \widehat{u}(t) \quad \text { for } t \in(0,1) .
$$


Also for $v \in D_{\bar{u}_{n}}^{\hat{u}_{n}}$ we have

$$
\begin{aligned}
&-\bar{u}_{n}^{\prime \prime}(t)+\gamma(t) \bar{u}_{n}(t) \\
&=-g_{1}\left(t, \bar{u}_{n}\right)+\lambda h_{1}\left(t, \bar{u}_{n}\right)+\gamma(t) \bar{u}_{n}(t) \\
& \leq-g_{1}(t, v)+\lambda h_{1}(t, v)+\gamma(t) v(t) \\
& \leq-g_{1}\left(t, \frac{1}{n}+v\right)+\lambda h_{1}(t, v)+\gamma(t) v(t) \\
& \leq g\left(t, \frac{1}{n}+v\right)+\lambda h(t, v)+\gamma(t) v(t) \quad \text { for } t \in(0,1), \\
&-\widehat{u}_{n}^{\prime \prime}(t)+\gamma(t) \widehat{u}_{n}(t) \\
& \geq g_{2}\left(t, \frac{1}{n}+\widehat{u}_{n}\right)+\lambda h_{1}(t, \bar{u})+\lambda h_{2}\left(t, \widehat{u}_{n}\right)+\gamma(t) \widehat{u}_{n}(t) \\
& \geq g_{2}\left(t, \frac{1}{n}+\widehat{u}_{n}\right)+\gamma(t) \widehat{u}_{n}(t)+\lambda h_{2}\left(t, \widehat{u}_{n}\right) \\
& \geq g_{2}\left(t, \frac{1}{n}+v\right)+\gamma(t) v(t)+\lambda h_{2}(t, v(t)) \\
& \geq g\left(t, \frac{1}{n}+v\right)+\lambda h(t, v)+\gamma(t) v(t) \quad \text { for } t \in(0,1) .
\end{aligned}
$$

Now Lemma 2.2 with $\delta_{n} \equiv 0, n \in N$ guarantees that there exists a solution $u \in C[0,1]$ to $(1.4)$ with

$$
\bar{u}(t) \leq u(t) \leq \widehat{u}(t) \quad \text { for } t \in[0,1] .
$$

\subsection{The Proof of Theorem 1.2}

Let

$$
\Lambda=\{\lambda \in R \mid \text { (1.4) has at least one positive solution }\} .
$$

Claim 2. Let

$$
\lambda^{*}=\frac{1}{\max _{t \in[0,1]} \int_{0}^{1} N(t, s) h_{2}\left(s, a_{2}+\phi_{1}\right) d s}>0 ;
$$


here

$$
\begin{array}{r}
a_{2}=1+\frac{1}{4} \int_{0}^{1}\left[g_{2}(t, 1)+\frac{1}{(\underline{\underline{u}}(t))^{\beta}}+e(t)\right] d t, \\
\quad \underline{\underline{u}}(t)=c_{2} l(t) \quad \text { for } t \in[0,1], \\
e(t)= \begin{cases}\sup _{r \in\left[c_{1}, 1+c_{2} / 2\right]} g^{-}(t, r), & \text { if } c_{1}<1+\frac{c_{2}}{2}, \\
0, & \text { if } c_{1} \geq 1+\frac{c_{2}}{2} .\end{cases}
\end{array}
$$

Then $\left(0, \lambda^{*}\right) \in \Lambda$.

Proof of Claim 2. Let $n \geq 1$ be fixed. Lemma 2.8 [6] implies that there exists $\alpha_{n, 1} \in C[0,1]$ such that

$$
\begin{gathered}
\underline{\underline{u}}(t) \leq \alpha_{n, 1}(t) \leq \overline{\bar{u}}(t) \\
-\alpha_{n, 1}^{\prime \prime}(t)=\bar{g}_{1}\left(t, \frac{1}{n}+\alpha_{n, 1}(t)\right)+\delta_{n}(t) \quad \text { for } t \in(0,1), \\
\alpha_{n, 1}(0)=\alpha_{n, 1}(1)=0
\end{gathered}
$$

where $\bar{g}_{1}$ is defined in (G5), and

$$
\begin{gathered}
\delta_{n}(t)=\bar{g}_{1}(t, \underline{\underline{u}}(t))-\bar{g}_{1}\left(t, \frac{1}{n}+\underline{\underline{u}}(t)\right), \\
\overline{\bar{u}}(t)=\bar{c} l(t) \quad \text { for } t \in[0,1] \\
\bar{c}=\max \left\{c_{1}, \pi \sup _{t \in(0,1)}\left[2 B\left(\frac{1}{(\underline{\underline{u}}(\cdot))^{\beta}}\right)(t)+B(e)(t)\right]\right\} .
\end{gathered}
$$

which does not depend on $n$.

On the other hand, let

$$
\psi(t, r)=g_{2}(t, r)+\frac{1}{\underline{\underline{u}}(t)^{\beta}}+e(t) .
$$


From (G1) notice $\psi$ satisfies the assumptions of Lemma 2.3, so there exist $\omega, \omega_{n} \in$ $C[0,1]$ such that

$$
\begin{gathered}
-\omega_{n}^{\prime \prime}(t)=g_{2}\left(t, \frac{1}{n}+\omega_{n}\right)+\frac{1}{\underline{\underline{u}}(t)^{\beta}}+e(t) \quad \text { for } t \in(0,1), \\
\omega_{n}(0)=\omega_{n}(1)=0, \\
\omega_{n}(t) \leq \omega_{n+1}(t) \leq 1+\omega_{1}(t) \leq a_{2} \text { for } t \in[0,1], n \in N, \\
\omega(t)=\lim _{n \rightarrow \infty} \omega_{n}(t) \quad \text { for } t \in[0,1], \\
-\omega^{\prime \prime}(t)=g_{2}(t, \omega(t))+\frac{1}{\underline{u}(t)^{\beta}}+e(t) \quad \text { for } t \in(0,1), \\
\omega(0)=\omega(1)=0 .
\end{gathered}
$$

Next we consider the boundary value problem

$$
\begin{gathered}
-\widetilde{v}_{n}^{\prime \prime}(t)=\lambda h_{2}\left(t, \omega_{n}+\widetilde{v}_{n}\right) \quad \text { for } t \in(0,1), \\
\widetilde{v}_{n}(0)=\widetilde{v}_{n}(1)=0,
\end{gathered}
$$

where $\lambda \in\left(0, \lambda^{*}\right)$.

Let $\Phi: C[0,1] \rightarrow C[0,1]$ be the operator defined by

$$
(\Phi v)(t):=\lambda \int_{0}^{1} G(t, s) h_{2}\left(s, \omega_{n}+v\right) d s \quad \text { for } v \in C[0,1], t \in[0,1]
$$

It is easy to see that $\Phi$ is a continuous and completely continuous operator. Also, if $0 \leq v(t) \leq$ $\phi_{1}(t)$ for $t \in[0,1]$, then

$$
\begin{aligned}
0 & \leq \Phi(v)(t)=\lambda \int_{0}^{1} G(t, s) h_{2}\left(s, \omega_{n}+v\right) d s \\
& \leq \lambda^{*} \int_{0}^{1} G(t, s) h_{2}\left(s, a_{2}+\phi_{1}\right) d s \\
& =\frac{\phi_{1}(t) \int_{0}^{1} N(t, s) h_{2}\left(s, a_{2}+\phi_{1}\right) d s}{\max _{t \in[0,1]} \int_{0}^{1} N(t, s) h_{2}\left(s, a_{2}+\phi_{1}\right) d s} \\
& \leq \phi_{1}(t) \quad \text { for } t \in[0,1] .
\end{aligned}
$$


Thus Schauder fixed point theorem guarantees that there exists $\widetilde{v}_{n} \in\left[0, \phi_{1}\right]$ such that $\Phi\left(\widetilde{v}_{n}\right)=$ $\widetilde{v}_{n}$, that is,

$$
\begin{gathered}
-\widetilde{v}_{n}^{\prime \prime}(t)=\lambda h_{2}\left(t, \omega_{n}+\widetilde{v}_{n}\right), \\
\widetilde{v}_{n}(0)=\widetilde{v}_{n}(1)=0 .
\end{gathered}
$$

Let

$$
\widehat{u}_{n}(t)=\omega_{n}(t)+\widetilde{v}_{n}(t), \quad \widehat{u}(t)=\omega(t)+\phi_{1}(t) \quad \text { for } t \in[0,1]
$$

Then $\widehat{u}_{n}, \widehat{u} \in C[0,1], \widehat{u}_{n}(0)=\widehat{u}_{n}(1)=0, \widehat{u}(0)=\widehat{u}(1)=0$,

$$
\begin{aligned}
0 & \leq \widehat{u}_{n}(t) \leq \widehat{u}(t) \quad \text { for } t \in[0,1] \\
-\widehat{u}_{n}^{\prime \prime}(t) & =-\omega_{n}^{\prime \prime}(t)-\widetilde{v}_{n}(t) \\
& =g_{2}\left(t, \frac{1}{n}+\omega_{n}\right)+\frac{1}{\underline{\underline{u}(t)^{\beta}}}+e(t)+\lambda h_{2}\left(t, \omega_{n}+\widetilde{v}_{n}\right) \\
& \geq g_{2}\left(t, \frac{1}{n}+\widehat{u}_{n}\right)+\frac{1}{\underline{\underline{u}}(t)^{\beta}}+e(t)+\lambda h_{2}\left(t, \widehat{u}_{n}\right) \quad \text { for } t \in(0,1), \lambda \in\left(0, \lambda^{*}\right) .
\end{aligned}
$$

Now let us consider the problem

$$
\begin{gathered}
-u^{\prime \prime}(t)=g\left(t, \frac{1}{n}+u\right)+\lambda h(t, u)+\delta_{n}(t) \quad \text { for } t \in(0,1), \lambda \in\left(0, \lambda^{*}\right) \\
u(0)=u(1)=0,
\end{gathered}
$$

where $\delta_{n}$ is defined in (2.47).

We will prove $\alpha_{n, 1}$ is a lower solution of (2.58) and $\widehat{u}_{n}$ is an upper solution of (2.58).

Now (2.46) and the positivity of $h(t, s)$ implies that

$$
\begin{aligned}
-\alpha_{n, 1}^{\prime \prime}(t) & =\bar{g}_{1}\left(t, \frac{1}{n}+\alpha_{n, 1}(t)\right)+\delta_{n}(t) \\
& \leq g\left(t, \frac{1}{n}+\alpha_{n, 1}(t)\right)+\lambda h\left(t, \alpha_{n, 1}(t)\right)+\delta_{n}(t),
\end{aligned}
$$


so $\alpha_{n, 1}$ is a lower solution of (2.58). On the other hand, from the definition of $\bar{g}_{1}$ and $\underline{\underline{u}}$, we have

$$
\begin{aligned}
\bar{g}_{1}(t, \underline{\underline{u}}) & =\min \left\{g(t, \underline{\underline{u}}), \frac{1}{\underline{\underline{u}}(t)^{\beta}}\right\} \leq \frac{1}{\underline{\underline{u}}(t)^{\beta}} \text { for } t \in(0,1), \\
-\bar{g}_{1}\left(t, \frac{1}{n}+\underline{\underline{u}}\right) & =-\min \left\{g^{+}\left(t, \frac{1}{n}+\underline{\underline{u}}\right), \frac{1}{(1 / n+\underline{\underline{u}})^{\beta}}\right\}+g^{-}\left(t, \frac{1}{n}+\underline{\underline{u}}\right) \\
& \leq g^{-}\left(t, \frac{1}{n}+\underline{\underline{u}}\right) \\
& \leq e(t) \quad \text { for } t \in(0,1),
\end{aligned}
$$

so

$$
\delta_{n}(t) \leq \frac{1}{\underline{\underline{u}}(t)^{\beta}}+e(t) \quad \text { for } t \in(0,1)
$$

Consequently, we have

$$
\begin{aligned}
-\widehat{u}_{n}^{\prime \prime}(t) & \geq g_{2}\left(t, \frac{1}{n}+\widehat{u}_{n}\right)+\frac{1}{\underline{\underline{u}}(t)^{\beta}}+e(t)+\lambda h_{2}\left(t, \widehat{u}_{n}\right) \\
& \geq g\left(t, \frac{1}{n}+\widehat{u}_{n}\right)+\frac{1}{\underline{\underline{u}}(t)^{\beta}}+e(t)+\lambda h\left(t, \widehat{u}_{n}\right) \\
& \geq g\left(t, \frac{1}{n}+\widehat{u}_{n}\right)+\lambda h\left(t, \widehat{u}_{n}\right)+\delta_{n}(t),
\end{aligned}
$$

so $\widehat{u}_{n}$ is an upper solution of (2.58). We next prove that

$$
\alpha_{n, 1}(t) \leq \widehat{u}_{n}(t) \quad \text { for } t \in[0,1]
$$

Suppose (2.63) is not true. Let $y(t)=\alpha_{n, 1}(t)-\widehat{u}_{n}(t)$ and let $\sigma \in(0,1)$ be the point where $y(t)$ attains its maximum over $(0,1)$. We have

$$
y(\sigma)>0, \quad y^{\prime \prime}(\sigma) \leq 0 .
$$


On the other hand, since $\alpha_{n, 1}(\sigma)>\widehat{u}_{n}(\sigma)$, we have

$$
\begin{aligned}
-\alpha_{n, 1}^{\prime \prime}(\sigma) & =\bar{g}_{1}\left(\sigma, \frac{1}{n}+\alpha_{n, 1}(\sigma)\right)+\delta_{n}(\sigma) \\
& \leq g\left(\sigma, \frac{1}{n}+\alpha_{n, 1}(\sigma)\right)+\delta_{n}(\sigma) \\
& \leq g\left(\sigma, \frac{1}{n}+\alpha_{n, 1}(\sigma)\right)+\frac{1}{\underline{\underline{u}}(\sigma)^{\beta}}+e(\sigma) \\
& \leq g_{2}\left(\sigma, \frac{1}{n}+\alpha_{n, 1}(\sigma)\right)+\frac{1}{\frac{\underline{u}}{=}(\sigma)^{\beta}}+e(\sigma) \\
& <g_{2}\left(\sigma, \frac{1}{n}+\widehat{u}_{n}(\sigma)\right)+\frac{1}{\underline{u}}(\sigma)^{\beta}+e(\sigma)+\lambda h_{2}\left(\sigma, \widehat{u}_{n}(\sigma)\right) \\
& \leq-\widehat{u}_{n}^{\prime \prime}(\sigma)
\end{aligned}
$$

so

$$
y^{\prime \prime}(\sigma)=\alpha_{n, 1}^{\prime \prime}(\sigma)-\widehat{u}_{n}^{\prime \prime}(\sigma)>0
$$

and this is a contradiction.

From (G3), there exists $\gamma \in M$ such that $r \rightarrow g(t, 1 / n+r)+\gamma(t) r$ is increasing in $\left(0,|\widehat{u}|_{\infty}\right)$. Let $\bar{u}(t) \equiv \underline{\underline{u}}(t), \bar{u}_{n}(t)=\alpha_{n, 1}(t)$. From (2.45), (2.56), and (2.63), we have

$$
0<\bar{u}(t) \leq \bar{u}_{n}(t) \leq \widehat{u}_{n}(t) \leq \widehat{u}(t) \text { for } t \in(0,1)
$$

Also for $v \in D_{\bar{u}_{n}}^{\widehat{u}_{n}}$, we have

$$
\begin{aligned}
-\bar{u}_{n}^{\prime \prime}(t) & +\gamma(t) \bar{u}_{n}(t) \\
\leq & g\left(t, \frac{1}{n}+\bar{u}_{n}\right)+\gamma(t) \bar{u}_{n}+\delta_{n}(t) \\
\leq & g\left(t, \frac{1}{n}+v\right)+\gamma(t) v+\delta_{n}(t)+\lambda h(t, v) \\
\leq & g\left(t, \frac{1}{n}+\widehat{u}_{n}\right)+\gamma(t) \widehat{u}_{n}+\delta_{n}(t)+\lambda h_{2}\left(t, \widehat{u}_{n}\right) \\
\leq & -\widehat{u}_{n}^{\prime \prime}(t)+\gamma(t) \widehat{u}_{n}(t)
\end{aligned}
$$


On the other hand, by (2.61)

$$
\begin{aligned}
& \left|\delta_{n}(t)\right| \leq \frac{1}{\bar{u}(t)^{\beta}}+e(t) \equiv \delta(t) \\
& \lim _{n \rightarrow \infty} \delta_{n}(t)=0 \quad \text { for } t \in(0,1) .
\end{aligned}
$$

Now Lemma 2.2 guarantees that there exists a solution $u \in C[0,1] \cap C^{1}(0,1)$ to (1.4) with

$$
\bar{u}(t) \leq u(t) \leq \widehat{u}(t) \quad \text { for } t \in[0,1] .
$$

Thus (1.4) has a solution for $\lambda \in\left(0, \lambda^{*}\right)$ so Claim 2 holds. In particular, $\Lambda \neq \emptyset$ and $\sup \Lambda>$ 0 .

Claim 3. If $\lambda \in \Lambda$, then $(0, \lambda] \in \Lambda$.

Proof of Claim 3.

Step 1. We may assume that $\lambda>0$. Let $x$ be a positive solution of (1.4), that is,

$$
\begin{gathered}
-x^{\prime \prime}=g(t, x)+\lambda h(t, x), \quad t \in(0,1), \\
x(0)=0=x(1) .
\end{gathered}
$$

We prove that there exists $\rho>0$ such that

$$
\chi(t) \geq \rho l(t) \text { for } t \in[0,1]
$$

By (G4), $g(t, r) \geq 0$ for $t \in(0,1), r \in\left(0, c_{1}\right]$. From the continuity of $\chi$ and $\chi(0)=0=\chi(1)$, it follows that there is $0<\delta<1 / 2$ such that

$$
0 \leq X(t)<c_{1} \quad \text { for } t \in[0, \delta] \cup[1-\delta, 1]
$$

Then

$$
-x^{\prime \prime} \geq \operatorname{lh}(t, x) \quad \text { for } t \in[0, \delta] \cup[1-\delta, 1]
$$

Let $v \in C^{1}(0, \delta) \cap C[0, \delta]$ so that

$$
\begin{gathered}
-v^{\prime \prime}(t)=h(t, X) \quad \text { for } t \in(0, \delta), \\
v(0)=v(\delta)=0 .
\end{gathered}
$$

It follows that $\lambda v(t) \leq \chi(t)$ for $t \in[0, \delta]$. Lemma 2.6 implies that there exists $m>0$ so that

$$
\operatorname{minf}\{t, \delta-t\} \leq v(t) \quad \text { for } t \in[0, \delta] .
$$


The same reason implies that

$$
m \inf \{t+\delta-1,1-t\} \leq v(t) \quad \text { for } t \in[1-\delta, 1]
$$

It follows that

$$
m \lambda l(t) \leq \chi(t) \quad \text { for } t \in\left[0, \frac{\delta}{2}\right] \cup\left[\frac{1-\delta}{2}, 1\right]
$$

Moreover,

$$
\inf \left\{\frac{x(t)}{l(t)}: t \in\left(0, \frac{\delta}{2}\right) \cup\left(\frac{1-\delta}{2}, 1\right)\right\}>0
$$

On the other hand, we easily have

$$
\inf \left\{\frac{X(t)}{l(t)}: t \in\left[\frac{\delta}{2}, \frac{1-\delta}{2}\right]\right\}>0
$$

so

$$
\inf \left\{\frac{x(t)}{l(t)}: t \in(0,1)\right\}=\rho>0
$$

and thus

$$
X(t) \geq \rho l(t) \quad \text { for } t \in[0,1] .
$$

Step 2. Let $\underline{\underline{r}}=\rho \wedge c_{2}$ and $\underline{\underline{u}}(t)=\underline{\underline{r}} l(t)$. Then

$$
\underline{\underline{u}}(t) \leq A\left(\bar{g}_{m}\left(\cdot, \frac{1}{n}+\underline{\underline{u}} \wedge \chi\right)+\delta_{n}\right)(t) \quad \text { for } t \in[0,1], m, n \geq 1,
$$

where

$$
\delta_{n}(t)=\bar{g}_{1}(t, \underline{\underline{u}} \wedge X)-\bar{g}_{1}\left(t, \frac{1}{n}+\underline{\underline{u}} \wedge X\right) .
$$

Notice

$$
\underline{\underline{u}}(t) \leq x(t), \quad \underline{\underline{u}}(t) \leq c_{2} l(t) \quad \text { for } t \in[0,1]
$$


From (G5), we have

$$
\begin{aligned}
A\left(\bar{g}_{1}(\cdot \underline{\underline{u}} \wedge x)\right)(t) & =\int_{0}^{1} G(t, s) \bar{g}_{1}(s, \underline{\underline{u}}) d s \\
& =\phi_{1}(t) \int_{0}^{1} N(t, s) \bar{g}_{1}(s, \underline{u}=d s \\
& \geq \frac{\phi_{1}(t)}{2 \pi} \int_{0}^{1} s(1-s) \bar{g}_{1}(s, \underline{r} \underline{\underline{r}}(s)) d s \\
& \geq \frac{\underline{\underline{r}} \phi_{1}(t)}{2} \geq \underline{\underline{u}}(t) \quad \text { for } t \in[0,1],
\end{aligned}
$$

so

$$
\begin{aligned}
& A\left(\bar{g}_{m}\left(\cdot, \frac{1}{n}+\underline{\underline{u}} \wedge x\right)+\delta_{n}\right)(t) \\
& \quad=\int_{0}^{1} G(t, s)\left[\bar{g}_{m}\left(s, \frac{1}{n}+\underline{\underline{u}} \wedge x\right)-\bar{g}_{1}\left(s, \frac{1}{n}+\underline{\underline{u}} \wedge x\right)+\bar{g}_{1}(s, \underline{\underline{u}} \wedge x)\right] d s \\
& \quad \geq \int_{0}^{1} G(t, s) \bar{g}_{1}(s, \underline{\underline{u}} \wedge x) d s \\
& \quad \geq \underline{\underline{u}}(t) \quad \text { for } t \in[0,1] .
\end{aligned}
$$

Step 3. Let $0<\mu<\lambda$. For each $m \geq 1$, there exists $\overline{\bar{r}}_{m}>\underline{\underline{r}}$, independent of $n$. Let

$$
\overline{\bar{u}}_{m}(t)=\overline{\bar{r}}_{m} l(t) \quad \text { for } t \in[0,1]
$$

Then

$$
A\left(\bar{g}_{m}\left(\cdot, \frac{1}{n}+v \wedge \chi\right)+\delta_{n}+\mu h_{2}(\cdot, v \wedge \chi)\right)(t) \leq \overline{\bar{u}}_{m}(t) \quad \text { for } t \in[0,1], v \in D_{\underline{\underline{u}}}^{\overline{\bar{u}}_{m}}, n \geq 1
$$

Let $v \in C[0,1] \cap C^{1}(0,1)$ such that

$$
\begin{gathered}
-v^{\prime \prime}=\lambda h_{2}(t, \chi) \quad \text { for } t \in(0,1), \\
v(0)=v(1)=0 .
\end{gathered}
$$

By Lemma 2.6, there exists $M>0$ such that

$$
v(t) \leq M l(t) \quad \text { for } t \in[0,1]
$$


Let

$$
\overline{\bar{r}}_{m}>\max \left\{M+\pi \sup _{t \in(0,1)} B\left(\frac{m}{(\underline{\underline{u}} \wedge \chi)^{\beta}}+\frac{1}{\underline{\underline{u^{\beta}}}}+e\right)(t), \underline{\underline{r}}\right\}
$$

Note $\underline{\underline{u}} \leq \overline{\bar{u}}$ since $\overline{\bar{r}}_{m}>\underline{\underline{r}}$. Let $v \geq \underline{\underline{u}}$ and notice (note $g^{-}(\cdot, r)=0$ if $0<r<c_{1}$ from (G4))

$$
\begin{aligned}
& A\left(\bar{g}_{m}\left(\cdot, \frac{1}{n}+v \wedge x\right)+\delta_{n}\right)(t) \\
& \quad=\int_{0}^{1} G(t, s)\left[\bar{g}_{m}\left(s, \frac{1}{n}+v \wedge x\right)-\bar{g}_{1}\left(s, \frac{1}{n}+\underline{\underline{u}} \wedge x\right)+\bar{g}_{1}(s, \underline{\underline{u}} \wedge x)\right] d s \\
& \quad \leq \int_{0}^{1} G(t, s)\left[\frac{m}{(1 / n+v \wedge x)^{\beta}}+g^{-}\left(s, \frac{1}{n}+\underline{\underline{u}}\right)+\bar{g}_{1}(s, \underline{u})\right] d s \\
& \quad \leq \int_{0}^{1} G(t, s)\left[\frac{m}{(v \wedge x)^{\beta}}+\frac{1}{\underline{u}^{\beta}}+e\right] d s \\
& \quad \leq \int_{0}^{1} G(t, s)\left[\frac{m}{(\underline{\underline{u}} \wedge x)^{\beta}}+\frac{1}{\underline{u^{\beta}}}+e\right] d s \\
& \quad=\phi_{1}(t)\left[B\left(\frac{m}{(\underline{\underline{u}} \wedge x)^{\beta}}+\frac{1}{\underline{\underline{u}}^{\beta}}+e\right)\right](t) \\
& \quad \leq \pi\left[B\left(\frac{m}{(\underline{\underline{u}} \wedge x)^{\beta}}+\frac{1}{\underline{u^{\beta}}}+e\right)\right](t) \cdot l(t) \quad \text { for } t \in[0,1] . \\
&
\end{aligned}
$$

On the other hand,

$$
\begin{aligned}
A(\mu h(\cdot, v \wedge \chi))(t) & =\mu \int_{0}^{1} G(t, s) h(s, v \wedge x) d s \\
& \leq \lambda \int_{0}^{1} G(t, s) h_{2}(s, x) d s \\
& =v(t) \leq M l(t) \text { for } t \in[0,1],
\end{aligned}
$$


so

$$
\begin{aligned}
A\left(\bar{g}_{m}\left(\cdot, \frac{1}{n}+v \wedge \chi\right)+\delta_{n}+\mu h(\cdot, v \wedge \chi)\right)(t) \\
\quad \leq A\left(\bar{g}_{m}\left(\cdot, \frac{1}{n}+v \wedge x\right)+\delta_{n}\right)(t)+A(\mu h(\cdot, v \wedge x))(t) \\
\quad \leq \pi\left[B\left(\frac{m}{(\underline{\underline{u}} \wedge x)^{\beta}}+\frac{1}{\underline{u}^{\beta}}+e\right)\right](t) \cdot l(t)+M l(t) \\
\leq \overline{\bar{u}}_{m}(t) \quad \text { for } t \in[0,1], v \in\left[\underline{\underline{u}}^{\prime} \overline{\bar{u}}_{m}\right], n \geq 1 .
\end{aligned}
$$

Step 4 . Let $0<\mu<\lambda$. Let $n, m \geq 1$ be fixed. There exists $\beta_{n, m} \in C[0,1]$ such that

$$
\begin{gathered}
\underline{\underline{u}}(t) \leq \beta_{n, m}(t) \leq \overline{\bar{u}}_{m}(t), \\
-\beta_{n, m}^{\prime \prime}(t)=\bar{g}_{m}\left(t, \frac{1}{n}+\beta_{n, m} \wedge x\right)+\mu h\left(t, \beta_{n, m} \wedge x\right)+\delta_{n}(t) \quad \text { for } t \in(0,1), \\
\beta_{n, m}(0)=\beta_{n, m}(1)=0 .
\end{gathered}
$$

Let $n, m>1$ be fixed. From Remark 1.3, there exist $\gamma_{n} \in M, \gamma_{n} \geq 0$ such that $\bar{g}_{m}(t, r)+$ $\gamma_{n}(t) r$ is increasing in $\left(1 / n, 1 / n+\overline{\bar{r}}_{m} / 2\right)$. We easily prove that

$$
\bar{g}_{m}(t, r \wedge \chi)+\gamma_{n}(t) r \text { is increasing in }\left(\frac{1}{n}, \frac{1}{n}+\frac{\overline{\bar{r}}_{m}}{2}\right) \text {. }
$$

Let $\bar{\gamma}(t)=\gamma_{n}$. We have $\bar{g}_{m}(t, 1 / n+r \wedge \chi)+\bar{\gamma}(t) r$ is increasing in $\left(0, \overline{\bar{r}}_{m} / 2\right)$. From (2.83) and (2.89), we have for fixed $v \in C[0,1], \underline{\underline{u}}(t) \leq v(t) \leq \overline{\bar{u}}_{m}(t)$ that

$$
\begin{aligned}
\underline{\underline{u}}(t)+A(\underline{\bar{\gamma}} \underline{\underline{u}})(t) & \leq A\left(\bar{g}_{m}\left(\cdot, \frac{1}{n}+\underline{\underline{u}} \wedge x\right)+\delta_{n}\right)(t)+A(\bar{r} \underline{\underline{u}})(t) \\
& \leq A\left(\bar{g}_{m}\left(\cdot, \frac{1}{n}+\underline{\underline{u}} \wedge x\right)+\bar{\gamma} \underline{\underline{u}}+\delta_{n}+\mu h(\cdot, v \wedge x)\right)(t) \\
& \leq A\left(\bar{g}_{m}\left(\cdot, \frac{1}{n}+v \wedge x\right)+\bar{\gamma} v+\delta_{n}+\mu h(\cdot, v \wedge x)\right)(t) \\
& \leq \overline{\bar{u}}_{m}(t)+A\left(\bar{\gamma} \overline{\bar{u}}_{m}\right)(t) .
\end{aligned}
$$


Fix $v \in C[0,1]$ with $\underline{\underline{u}}(t) \leq v(t) \leq \overline{\bar{u}}_{m}(t)$. From Lemma 2.4, there exists $\Psi(v) \in C[0,1]$ such that

$$
\begin{aligned}
& -\Psi^{\prime \prime}(v)(t)+\bar{\gamma}(t) \Psi(v)(t) \\
& \quad=\bar{g}_{m}\left(t, \frac{1}{n}+v \wedge X\right)+\bar{\gamma}(t) v(t)+\delta_{n}(t)+\mu h(t, v \wedge X) \quad \text { for } t \in(0,1) \\
& \Psi(v)(0)=\Psi(v)(1)=0
\end{aligned}
$$

Then

$$
\Psi(v)(t)+A(\bar{\gamma} \Psi(v))(t)=A\left(\bar{g}_{m}\left(\cdot, \frac{1}{n}+v \wedge \chi\right)+\bar{\gamma} v+\delta_{n}+\mu h(\cdot, v \wedge \chi)\right)(t) \quad \text { for } t \in(0,1),
$$

so (2.98) implies that

$$
\begin{aligned}
\underline{\underline{u}}(t)+A(\bar{\gamma} \underline{\underline{u}})(t) & \leq \Psi(v)(t)+A(\bar{\gamma} \Psi(v))(t) \\
& \leq \overline{\bar{u}}_{m}(t)+A\left(\bar{\gamma} \overline{\bar{u}}_{m}\right)(t) \quad \text { for } t \in(0,1) .
\end{aligned}
$$

From Corollary 2.5, we have

$$
\underline{\underline{u}}(t) \leq \Psi(v)(t) \leq \overline{\bar{u}}_{m}(t) \quad \text { for } t \in[0,1]
$$

Also,

$$
\begin{aligned}
& \left|\bar{g}_{m}\left(t, \frac{1}{n}+v \wedge x\right)+\bar{\gamma} v+\delta_{n}+\mu h(t, v \wedge x)\right| \\
& \quad \leq g_{1}\left(t, \frac{\phi_{1}(t)}{n}\right)+g_{2}\left(t, \frac{1}{n}\right)+\bar{\gamma}\left|\overline{\bar{u}}_{m}\right|_{\infty}+\left|\delta_{n}(t)\right|+\lambda h_{2}\left(t,|x|_{\infty}\right) \\
& \quad \equiv \beta(t) \in M \quad \text { for } t \in(0,1) .
\end{aligned}
$$

Now $\Psi: D_{\underline{\underline{u}}}^{\overline{\bar{u}}_{m}} \rightarrow D_{\underline{\underline{u}}}^{\overline{\bar{u}}_{m}}$ is compact, so Schauder's fixed point theorem implies that there exists $\beta_{n, m} \in C[0, \overline{1}]$ such that $\underline{\underline{u}}(t) \leq \beta_{n, m}(t) \leq \overline{\bar{u}}_{m}(t)$ and $\Psi\left(\beta_{n, m}\right)(t)=\beta_{n, m}(t)$ for $t \in(0,1)$ :

$$
\begin{gathered}
-\beta_{n, m}^{\prime \prime}(t)=\bar{g}_{m}\left(t, \frac{1}{n}+\beta_{n, m} \wedge x\right)+\mu h\left(t, \beta_{n, m} \wedge X\right)+\delta_{n}(t) \quad \text { for } t \in(0,1), \\
\beta_{n, m}(0)=\beta_{n, m}(1)=0, \\
\left|\bar{g}_{m}\left(t, \frac{1}{n}+\beta_{n, m} \wedge X\right)+\mu h\left(t, \beta_{n, m} \wedge x\right)+\delta_{n}(t)\right| \leq 3 g_{2}(t, \underline{u} \wedge x)+\lambda h_{2}(t, x) .
\end{gathered}
$$


Let $m \geq 1$ be fixed. We consider the sequence $\left\{\beta_{n, m}\right\}_{n=1}^{\infty}$. Fix $n_{0} \in\{2,3, \ldots\}$. Let us look at the interval $\left[1 / 2^{n_{0}+1}, 1-1 / 2^{n_{0}+1}\right]$. The mean value theorem implies that there exists $\tau \in\left(1 / 2^{m_{0}+1}, 1-1 / 2^{m_{0}+1}\right)$ with $\left|\beta_{n, m}^{\prime}(\tau)\right| \leq(8 / 3) \sup _{t \in[0,1]} \overline{\bar{u}}_{m}(t)$. As a result

$$
\left\{\beta_{n, m}(t)\right\}_{n=n_{0}+1}^{\infty} \text { is bounded, equicontinuous family on }\left[\frac{1}{2^{n_{0}+1}}, 1-\frac{1}{2^{n_{0}+1}}\right] \text {. }
$$

The Arzela-Ascoli theorem guarantees the existence of subsequence $N_{n_{0}}$ of integers and a function $z_{n_{0}, m} \in\left[1 / 2^{n_{0}+1}, 1-1 / 2^{n_{0}+1}\right]$ with $\beta_{n, m}$ converging uniformly to $z_{n_{0}, m}$ on $\left[1 / 2^{n_{0}+1}, 1-\right.$ $1 / 2^{n_{0}+1}$ ] as $n \rightarrow \infty$ through $N_{n_{0}}$. Similarly,

$$
\left\{\beta_{n, m}\right\}_{n=n_{0}+1}^{\infty} \text { is bounded, equicontinuous family on }\left[\frac{1}{2^{n_{0}+2}}, 1-\frac{1}{2^{n_{0}+2}}\right] \text {, }
$$

so there is a subsequence $N_{n_{0}+1}$ of $N_{n_{0}}$ and a function $z_{n_{0}+1, m} \in C\left[1 / 2^{n_{0}+2}, 1-1 / 2^{n_{0}+2}\right]$ with $\beta_{n, m}$ converging uniformly to $z_{n_{0}+1, m}$ on $\left[1 / 2^{n_{0}+2}, 1-1 / 2^{n_{0}+2}\right]$ as $n \rightarrow \infty$ through $N_{n_{0}+1}$. Note $z_{n_{0}+1, m}=z_{n_{0}, m}$ on $\left[1 / 2^{n_{0}+1}, 1-1 / 2^{n_{0}+1}\right]$ since $N_{n_{0}+1} \subseteq N_{n_{0}}$. Proceed inductively to obtain subsequences of integers $N_{n_{0}} \supseteq N_{n_{0}+1} \supseteq \cdots \supseteq N_{k} \supseteq \cdots$ and functions $z_{k, m} \in C\left[1 / 2^{k+1}, 1-\right.$ $\left.1 / 2^{k+1}\right]$ with $\beta_{n, m}$ converging uniformly to $z_{k, m}$ on $\left[1 / 2^{k+1}, 1-1 / 2^{k+1}\right]$ as $n \rightarrow \infty$ through $N_{k}$ and $z_{k, m}=z_{k-1, m}$ on $\left[1 / 2^{k}, 1-1 / 2^{k}\right]$.

Define a function $u_{m}:[0,1] \rightarrow[0, \infty)$ by $u_{m}(t)=z_{k, m}(t)$ on $\left[1 / 2^{k+1}, 1-1 / 2^{k+1}\right]$ and $u_{m}(0)=u_{m}(1)=0$. Notice $u_{m}$ is well defined and $\underline{\underline{u}}(t) \leq u_{m}(t) \leq \overline{\bar{u}}_{m}(t)$ for $t \in(0,1)$. Next, fix $t \in(0,1)$ (without loss of generality assume $t \neq 1 / \overline{\overline{2}}$ ) and let $n^{*} \in\left\{n_{0}, n_{0}+1, \ldots\right\}$ be such that $1 / 2^{n^{*}+1}<t<1-1 / 2^{n^{*}+1}$. Let $N_{n^{*}}^{*}=\left\{i \in N_{n}: i \geq n^{*}\right\}$. Now $\beta_{n, m}, n \in N_{n^{*}}^{*}$ satisfies the integral equation

$$
\begin{aligned}
\beta_{n, m}(t)= & \beta_{n, m}\left(\frac{1}{2}\right)+\beta_{n, m}^{\prime}\left(\frac{1}{2}\right)\left(t-\frac{1}{2}\right) \\
& +\int_{1 / 2}^{t}(s-t)\left(\bar{g}_{m}\left(s, \frac{1}{n}+\beta_{n, m} \wedge x\right)+\mu h\left(s, \beta_{n, m} \wedge x\right)+\delta_{n}(s)\right) d s,
\end{aligned}
$$

for $t \in\left[1 / 2^{n+1}, 1-1 / 2^{n+1}\right]$. Notice (take $t=2 / 3$ say) that $\left\{\beta_{n, m}(1 / 2)\right\}, n \in N_{n^{*}}^{*}$, is a bounded sequence since $\underline{\underline{u}}(t) \leq \beta_{n, m}(t) \leq \overline{\bar{u}}_{m}(t)$ for $t \in[0,1]$. Thus $\left\{\beta_{n, m}(1 / 2)\right\}_{n \in N_{n^{*}}^{*}}$ has a convergent subsequence; for convenience we will let $\left\{\beta_{n, m}(1 / 2)\right\}_{n \in N_{n^{*}}^{*}}$ denote this subsequence also, and let $\tau \in R$ be its limit. Now for the above fixed $t$, and let $n \rightarrow \infty$ through $N_{n^{*}}^{*}$ to obtain

$$
\begin{aligned}
g_{m}\left(t, \frac{1}{n}+\beta_{n, m} \wedge x\right) & \longrightarrow g_{m}\left(t, z_{k, m} \wedge x\right), \\
h\left(t, \beta_{n, m} \wedge x\right) & \longrightarrow h\left(t, z_{k, m} \wedge x\right), \\
\delta_{n} & \longrightarrow 0 .
\end{aligned}
$$


As a result,

$$
z_{k, m}(t)=z_{k, m}\left(\frac{1}{2}\right)+\tau\left(t-\frac{1}{2}\right)+\int_{1 / 2}^{t}(s-t)\left(\bar{g}_{m}\left(s, z_{k, m} \wedge x\right)+\mu h\left(s, z_{k, m} \wedge x\right)\right) d s
$$

that is,

$$
u_{m}(t)=u_{m}\left(\frac{1}{2}\right)+\tau\left(t-\frac{1}{2}\right)+\int_{1 / 2}^{t}(s-t)\left(\bar{g}_{m}\left(s, u_{m} \wedge \chi\right)+\mu h\left(s, u_{m} \wedge \chi\right)\right) d s
$$

We can do this argument for each $t \in(0,1)$ and so

$$
-u_{m}^{\prime \prime}(t)=\bar{g}_{m}\left(t, u_{m} \wedge \chi\right)+\mu h\left(t, u_{m} \wedge \chi\right) \quad \text { for } t \in(0,1)
$$

It remains to show that $u_{m}$ is continuous at 0 and 1 .

Let $\varepsilon>0$ be given. Since $\overline{\bar{u}}_{m} \in C[0,1]$ there exists $\delta>0$ with $\overline{\bar{u}}_{m}(t)<\varepsilon / 2$ for $t \in[0, \delta]$. As a result $\underline{\underline{u}}(t) \leq \beta_{n, m}(t) \leq \overline{\bar{u}}_{m}(t)<\varepsilon / 2$ for $t \in[0, \delta]$. Consequently, $\underline{\underline{u}}(t) \leq u_{m}(t) \leq \varepsilon / 2<\varepsilon$ for $t \in[0, \delta]$ and so $u_{m}$ is continuous at 0 . Similarly, $u_{m}$ is continuous at $\overline{\overline{1}}$. As a result $u_{m} \in C[0,1]$ and

$$
\begin{gathered}
-u_{m}^{\prime \prime}(t)=\bar{g}_{m}\left(t, u_{m} \wedge \chi\right)+\mu h\left(t, u_{m} \wedge \chi\right) \quad \text { for } t \in(0,1) \\
u_{m}(0)=u_{m}(1)=0 .
\end{gathered}
$$

Next we prove

$$
u_{m}(t) \leq \chi(t) \text { for } t \in[0,1]
$$

Suppose (2.113) is not true. Let $y(t)=u_{m}(t)-x(t)$ and $\sigma \in(0,1)$ be the point where $y(t)$ attains its maximum over $(0,1)$. We have

$$
y(\sigma)>0, \quad y^{\prime \prime}(\sigma) \leq 0
$$

On the other hand, since $u_{m}(\sigma)>\chi(\sigma)$, we have

$$
\begin{aligned}
y^{\prime \prime}(\sigma) & =u_{m}^{\prime \prime}(\sigma)-\chi^{\prime \prime}(\sigma) \\
& =-\bar{g}_{m}\left(\sigma, u_{m} \wedge \chi\right)-\mu h\left(\sigma, u_{m} \wedge \chi\right)+g(\sigma, \chi)+\lambda h(\sigma, \chi) \\
& =-\bar{g}_{m}(\sigma, \chi(\sigma))-\mu h(\sigma, \chi(\sigma))+g(\sigma, \chi(\sigma))+\lambda h(\sigma, \chi(\sigma)) \\
& \geq(\lambda-\sigma) h(\sigma, \chi(\sigma))>0 .
\end{aligned}
$$

This is a contradiction, so (2.113) is true. 
Thus we have

$$
\begin{gathered}
-u_{m}^{\prime \prime}=g_{m}\left(t, u_{m}\right)+\mu h\left(t, u_{m}\right), \\
u_{m}(0)=u_{m}(1)=0, \\
\underline{\underline{u}}(t) \leq u_{m}(t) \leq \chi(t) \quad \text { for } t \in[0,1] .
\end{gathered}
$$

By the same reason as above, we obtain subsequences of integers $N_{m_{0}} \supseteq N_{m_{0}+1} \supseteq \cdots \supseteq$ $N_{k} \supseteq \cdots$ and functions $z_{m} \in C\left[1 / 2^{k+1}, 1-1 / 2^{k+1}\right]$ with $u_{m}$ converging uniformly to $z_{k}$ on $\left[1 / 2^{k+1}, 1-1 / 2^{k+1}\right]$ as $m \rightarrow \infty$ through $N_{k}$, and $z_{k}=z_{k-1}$ on $\left[1 / 2^{k}, 1-1 / 2^{k}\right]$.

Define a function $u:[0,1] \rightarrow[0, \infty)$ by $u(t)=z_{k}(t)$ on $\left[1 / 2^{k+1}, 1-1 / 2^{k+1}\right]$ and $u(0)=$ $u(1)=0$. Notice $u$ is well defined and $\underline{\underline{u}}(t) \leq u(t) \leq \chi(t)$ for $t \in(0,1)$. Next fix $t \in(0,1)$ (without loss of generality assume $t \neq 1 / 2$ ) and let $m^{*} \in\left\{m_{0}, m_{0}+1, \ldots\right\}$ be such that $1 / 2^{m^{*}+1}<$ $t<1-1 / 2^{m^{*}+1}$. Let $N_{m^{*}}^{*}=\left\{k \in N_{m^{*}}: k \geq m^{*}\right\}$. Now $u_{m}, m \in N_{m^{*}}^{*}$ satisfies the integral equation

$$
u_{m}(t)=u_{m}\left(\frac{1}{2}\right)+u_{m}^{\prime}\left(\frac{1}{2}\right)\left(t-\frac{1}{2}\right)+\int_{1 / 2}^{t}(s-t)\left(\bar{g}_{m}\left(s, u_{m}\right)+\mu h\left(s, u_{m}\right)\right) d s
$$

for $t \in\left[1 / 2^{m^{*}+1}, 1-1 / 2^{m^{*}+1}\right]$. Notice (take $t=2 / 3$ say) that $\left\{u_{m}(1 / 2)\right\}, m \in N_{m^{*}}^{*}$ is a bounded sequence since $\underline{\underline{u}}(t) \leq u_{m}(t) \leq \chi(t)$ for $t \in[0,1]$. Thus $\left\{u_{m}(1 / 2)\right\}_{m \in N_{m^{*}}^{*}}$ has a convergent subsequence; for convenience we will let $\left\{u_{m}(1 / 2)\right\}_{m \in N_{m^{*}}^{*}}$ denote this subsequence also, and let $\tau \in R$ be its limit. Now for the above fixed $t$, and letting $m \rightarrow \infty$ through $N_{k}^{*}$ to obtain

$$
u(t)=u\left(\frac{1}{2}\right)+\tau\left(t-\frac{1}{2}\right)+\int_{1 / 2}^{t}(s-t)(g(s, u)+\mu h(s, u)) d s
$$

we can do this argument for each $t \in(0,1)$ and so

$$
-u^{\prime \prime}(t)=g(t, u)+\mu h(t, u) \quad \text { for } t \in(0,1)
$$

Also reasoning as before we have that $u$ is continuous at 0 and 1.

Thus we have

$$
\begin{gathered}
-u^{\prime \prime}=g(t, u)+\mu h(t, u), \\
u(0)=u(1)=0 .
\end{gathered}
$$

Now let $\lambda_{2}^{*}=\sup \Lambda>0$. Then

(i) if $0<\lambda<\lambda_{2}^{*}$, (1.4) has at least one solution $u \in C[0,1] \cap C^{1}(0,1)$ and $u>0$ for $t \in(0,1)$;

(ii) if $\lambda>\lambda_{2}^{*}$, (1.4) has no solutions. 


\subsection{The Proof of Theorem 1.4}

Claim 4. Let

$$
\lambda^{*}=\frac{1}{\max _{t \in[0,1]} \int_{0}^{1} N(t, s) h_{2}\left(s, a_{3}+\phi_{1}\right) d s}>0 ;
$$

here

$$
a_{3}=1+\frac{1}{4} \int_{0}^{1}\left(g_{2}(s, 1)+h_{2}\left(s, \frac{1}{2}+\phi_{1}(s)\right)+\frac{\phi_{1}(s)}{2\left|\phi_{1}\right|_{\infty}}\right) d s .
$$

Then $\left(0, \lambda^{*}\right) \in \Lambda$.

Proof of Claim 4. Let $\lambda \in\left(0, \lambda^{*}\right)$ be fixed. From assumption (G6), it follows that there is $\tau \geq \tau_{1}$ and $c_{3} \in(0,1)$, such that if $n>2 / c_{3}, 0<k<c_{3} / 2<1$, we have

$$
\begin{aligned}
& 0<k\left|\phi_{1}\right|_{\infty}<\frac{c_{3}}{2}, \quad 0<\frac{1}{n}+k \phi_{1}(t)<c_{3} \\
& \frac{\tau\left(1 / n+k \phi_{1}(t)\right)+g^{-}\left(t, 1 / n+k \phi_{1}(t)\right)}{h\left(t, 1 / n+k \phi_{1}(t)\right)} \leq \lambda .
\end{aligned}
$$

Thus,

$$
\frac{\tau k \phi_{1}(t)+g^{-}\left(t, 1 / n+k \phi_{1}(t)\right)}{h\left(t, 1 / n+k \phi_{1}(t)\right)} \leq \lambda
$$

Then, for $n>2 / c_{3}$,

$$
\tau k \phi_{1}(t)+g^{-}\left(t, \frac{1}{n}+k \phi_{1}(t)\right) \leq \lambda h\left(t, \frac{1}{n}+k \phi_{1}(t)\right)
$$

and we have

$$
\begin{aligned}
\tau k \phi_{1}(t) \leq & \lambda h\left(t, \frac{1}{n}+k \phi_{1}(t)\right)-g^{-}\left(t, \frac{1}{n}+k \phi_{1}(t)\right) \\
\leq & g^{+}\left(t, \frac{1}{n}+k \phi_{1}(t)\right)-g^{-}\left(t, \frac{1}{n}+k \phi_{1}(t)\right)+\lambda h\left(t, \frac{1}{n}+k \phi_{1}(t)\right) \\
= & g\left(t, \frac{1}{n}+k \phi_{1}(t)\right)+\lambda h\left(t, \frac{1}{n}+k \phi_{1}(t)\right) \\
& -\lambda h\left(t, k \phi_{1}(t)\right)+\lambda h\left(t, k \phi_{1}(t)\right) \\
= & g\left(t, \frac{1}{n}+k \phi_{1}(t)\right)+\lambda h\left(t, k \phi_{1}(t)\right)+\delta_{n}(t),
\end{aligned}
$$


where

$$
\delta_{n}(t)=\lambda h\left(t, \frac{1}{n}+k \phi_{1}(t)\right)-\lambda h\left(t, k \phi_{1}(t)\right)
$$

Let $\bar{u}(t)=k \phi_{1}(t)$. We have

$$
-\bar{u}^{\prime \prime}(t)=\tau_{1} k \phi_{1}(t) \leq \tau k \phi_{1}(t) \leq g\left(t, \frac{1}{n}+\bar{u}(t)\right)+\lambda h(t, \bar{u}(t))+\delta_{n}(t) \quad \text { for } t \in(0,1) .
$$

Let

$$
\psi(t, s)=g_{2}(t, s)+\lambda h_{2}\left(t, \frac{1}{2}+\phi_{1}(t)\right)+\frac{\phi_{1}(t)}{2\left|\phi_{1}\right|_{\infty}} .
$$

From (G1) notice that $\psi$ satisfies the assumptions of Lemma 2.3, so there exist $\omega, \omega_{n} \in$ $C[0,1]$ such that

$$
\begin{gathered}
-\omega_{n}^{\prime \prime}(t)=g_{2}\left(t, \frac{1}{n}+\omega_{n}\right)+\lambda h_{2}\left(t, \frac{1}{2}+\phi_{1}(t)\right)+\frac{\phi_{1}(t)}{2\left|\phi_{1}\right|_{\infty}} \text { for } t \in(0,1), \\
\omega_{n}(0)=\omega_{n}(1)=0, \\
\omega(t)=\lim _{n \rightarrow \infty} \omega_{n}(t) \quad \text { for } t \in[0,1], \\
\omega_{n}(t) \leq 1+\frac{1}{4} \int_{0}^{1}\left(g_{2}(s, 1)+h_{2}\left(s, \frac{1}{2}+\phi_{1}(s)\right)+\frac{\phi_{1}(s)}{2\left|\phi_{1}\right|_{\infty}}\right) d s=a_{3} \quad \text { for } t \in[0,1], n \in N .
\end{gathered}
$$

Consider the boundary value problem

$$
\begin{gathered}
-\widetilde{v}^{\prime \prime}(t)=\lambda h_{2}\left(t, \omega_{n}+\widetilde{v}\right) \quad \text { for } t \in(0,1), \\
\widetilde{v}(0)=\widetilde{v}(1)=0 .
\end{gathered}
$$

Let $\Phi: C[0,1] \rightarrow C[0,1]$ be the operator defined by

$$
(\Phi v)(t):=\lambda \int_{0}^{1} G(t, s) h_{2}\left(s, \omega_{n}+v\right) d s \quad \text { for } v \in C[0,1], t \in[0,1]
$$


It is easy to see that $\Phi$ is a continuous and completely continuous operator. Also if $0 \leq v(t) \leq$ $\phi_{1}(t)$ for $t \in[0,1]$, then

$$
\begin{aligned}
0 & \leq \Phi(v)(t)=\lambda \int_{0}^{1} G(t, s) h_{2}\left(s, \omega_{n}+v\right) d s \\
& \leq \lambda^{*} \int_{0}^{1} G(t, s) h_{2}\left(s, a_{3}+\phi_{1}\right) d s \\
& =\frac{\phi_{1}(t) \int_{0}^{1} N(t, s) h_{2}\left(s, a_{3}+\phi_{1}\right) d s}{\max _{t \in[0,1]} \int_{0}^{1} N(t, s) h_{2}\left(s, a_{3}+\phi_{1}\right) d s} \\
& \leq \phi_{1}(t) \quad \text { for } t \in[0,1]
\end{aligned}
$$

Thus Schauder's fixed point theorem guarantees that there exists $\widetilde{v}_{n} \in\left[0, \phi_{1}\right]$ such that $\Phi\left(\widetilde{v}_{n}\right)=\widetilde{v}_{n}$, that is,

$$
\begin{gathered}
-\widetilde{v}_{n}^{\prime \prime}(t)=\lambda h_{2}\left(t, \omega_{n}+\widetilde{v}_{n}\right), \\
\tilde{v}_{n}(0)=\widetilde{v}_{n}(1)=0 .
\end{gathered}
$$

Let

$$
\widehat{u}_{n}(t)=\omega_{n}(t)+\widetilde{v}_{n}(t), \quad \widehat{u}(t)=\omega(t)+\phi_{1}(t) \quad \text { for } t \in[0,1]
$$

Then $\widehat{u}_{n}, \widehat{u} \in C[0,1], \widehat{u}_{n}(0)=\widehat{u}_{n}(1)=0, \widehat{u}(0)=\widehat{u}(1)=0,0 \leq \widehat{u}_{n}(t) \leq \widehat{u}(t)$ for $t \in[0,1]$, and

$$
\begin{aligned}
-\widehat{u}_{n}^{\prime \prime}(t) & =-\omega_{n}^{\prime \prime}(t)-\widetilde{v}_{n}^{\prime \prime}(t) \\
& =g_{2}\left(t, \frac{1}{n}+\omega_{n}\right)+\lambda h_{2}\left(t, \frac{1}{2}+\phi_{1}(t)\right)+\frac{\phi_{1}(t)}{2\left|\phi_{1}\right|}+\lambda h_{2}\left(t, \omega_{n}+\widetilde{v}_{n}\right) \\
& \geq g_{2}\left(t, \frac{1}{n}+\widehat{u}_{n}\right)+\lambda h_{2}\left(t, \frac{1}{2}+\phi_{1}(t)\right)+\frac{\phi_{1}(t)}{2\left|\phi_{1}\right|}+\lambda h_{2}\left(t, \widehat{u}_{n}\right) \quad \text { for } t \in(0,1) .
\end{aligned}
$$

We next prove that

$$
\bar{u}(t) \leq \widehat{u}_{n}(t) \quad \text { for } t \in[0,1]
$$

Suppose (2.137) is not true. Let $y(t)=\bar{u}(t)-\widehat{u}_{n}(t)$ and $\sigma \in(0,1)$ be the point where $y(t)$ attains its maximum over $(0,1)$. We have

$$
y(\sigma)>0, \quad y^{\prime \prime}(\sigma) \leq 0
$$


On the other hand, since $\bar{u}(\sigma)>\widehat{u}_{n}(\sigma)$, we have

$$
\begin{aligned}
-\bar{u}^{\prime \prime}(\sigma) & \leq g\left(\sigma, \frac{1}{n}+\bar{u}(\sigma)\right)+\lambda h(\sigma, \bar{u}(\sigma))+\delta_{n}(\sigma) \\
& =g\left(\sigma, \frac{1}{n}+\bar{u}(\sigma)\right)+\lambda h\left(\sigma, \frac{1}{n}+\bar{u}(\sigma)\right) \\
& \leq g_{2}\left(\sigma, \frac{1}{n}+\bar{u}(\sigma)\right)+\lambda h_{2}\left(\sigma, \frac{1}{2}+\phi_{1}(\sigma)\right) \\
& <g_{2}\left(\sigma, \frac{1}{n}+\widehat{u}_{n}(\sigma)\right)+\lambda h_{2}\left(\sigma, \frac{1}{2}+\phi_{1}(\sigma)\right)+\frac{\phi_{1}(\sigma)}{2\left|\phi_{1}\right|}+\lambda h_{2}\left(\sigma, \widehat{u}_{n}(\sigma)\right) \\
& \leq-\widehat{u}_{n}^{\prime \prime}(\sigma) .
\end{aligned}
$$

Thus $y^{\prime \prime}(\sigma)=\bar{u}^{\prime \prime}(\sigma)-\widehat{u}_{n}^{\prime \prime}(\sigma)>0$, and this is a contradiction. As a result, (2.137) is true.

On the other hand, we have

$$
\begin{aligned}
\left|\delta_{n}(t)\right| & \leq \lambda\left|h\left(t, \frac{1}{n}+k \phi_{1}(t)\right)-h\left(t, k \phi_{1}(t)\right)\right| \\
& \leq 2 \lambda h_{2}\left(t, \frac{1}{2}+\left|\phi_{1}\right|\right)
\end{aligned}
$$

for $n>2 / c_{3}$. Consequently, for $t \in(0,1), \delta_{n} \rightarrow 0$ and $n \rightarrow \infty$.

From assumptions (G2) and (H5), there exists a $\gamma, \tau \in M, n>2 / c_{3}$, so that $g(t, 1 / n+$ $r)+h(t, r)+a(t) r$ is increasing in $\left(0,|\widehat{u}|_{\infty}\right)$, where $a(t)=\gamma(t)+\tau(t)$. Let $\bar{u}_{n}=\bar{u}(t)$. For $v \in D_{\bar{u}_{n}}^{\widehat{u}_{n}}$, we have

$$
\begin{aligned}
-\bar{u}_{n}^{\prime \prime}(t) & +a(t) \bar{u}_{n}(t) \\
\leq & g\left(t, \frac{1}{n}+\bar{u}_{n}(t)\right)+\lambda h\left(t, \bar{u}_{n}(t)\right)+\delta_{n}(t)+a(t) \bar{u}_{n}(t) \\
\leq & g\left(t, \frac{1}{n}+\bar{u}_{n}(t)\right)+\lambda h\left(t, \bar{u}_{n}(t)\right)+a(t) \bar{u}_{n}(t) \\
& +\lambda h\left(t, \frac{1}{n}+\bar{u}_{n}(t)\right)-\lambda h\left(t, \bar{u}_{n}(t)\right) \\
\leq & g\left(t, \frac{1}{n}+\widehat{u}_{n}(t)\right)+\lambda h\left(t, \widehat{u}_{n}(t)\right)+a(t) \widehat{u}_{n}(t)+\lambda h\left(t, \frac{1}{n}+\bar{u}_{n}(t)\right) \\
\leq & g_{2}\left(t, \frac{1}{n}+\widehat{u}_{n}(t)\right)+\lambda h_{2}\left(t, \widehat{u}_{n}(t)\right)+\frac{\phi_{1}(t)}{2\left|\phi_{1}\right|_{\infty}} \\
& +\lambda h_{2}\left(t, \frac{1}{2}+\phi_{1}(t)\right)+a(t) \widehat{u}_{n}(t) \\
\leq & -\widehat{u}_{n}^{\prime \prime}(t)+a_{n}(t) \widehat{u}_{n}(t) .
\end{aligned}
$$


Reasoning as in the proof of Theorem 1.1, Lemma 2.2 guarantees that (1.4) has a solution $u \in C[0,1] \cap C^{1}(0,1)$. $\sup \Lambda>0$.

Thus (1.4) has a solution for $\lambda \in\left(0, \lambda^{*}\right)$ so Claim 4 holds. In particular, $\Lambda \neq \emptyset$ and

Claim 5. If $\lambda \in \Lambda$, then $(0, \lambda] \in \Lambda$.

Proof of Claim 5. We may assume that $\lambda>0$. Let $x$ be a positive solution of (1.4), that is,

$$
\begin{gathered}
-x^{\prime \prime}=g(t, x)+\lambda h(t, x), \quad t \in(0,1), \\
x(0)=0=x(1) .
\end{gathered}
$$

We first prove that there exists $\rho>0$ such that

$$
x(t) \geq \rho l(t) \text { for } t \in[0,1]
$$

By (G6), there exists $\sigma>0$ such that for all $r \in(0, \sigma)$, we have

$$
\frac{\tau r+g^{-}(t, r)}{h(t, r)} \leq \lambda
$$

that is,

$$
\tau r \leq \lambda h(t, r)-g^{-}(t, r) \quad \text { for } t \in(0,1), r \in(0, \sigma) .
$$

From the continuity of $x$ and $\chi(0)=0=x(1)$, it follows that there is $0<\delta<1 / 2$ such that

$$
\chi(t)<\sigma \quad \text { for } t \in[0, \delta] \cup[1-\delta, 1]
$$

Then

$$
\begin{aligned}
-x^{\prime \prime} & =g(t, x)+\lambda h(t, x) \\
& =g^{+}(t, x)+\lambda h(t, x)-g^{-}(t, x) \\
& \geq \lambda h(t, x)-g^{-}(t, x) \\
& \geq \tau \chi(t) \quad \text { for } t \in[0, \delta] \cup[1-\delta, 1] .
\end{aligned}
$$

The next part is similar to the proof of (2.72), that is, there exists $\rho>0$ such that

$$
\chi(t) \geq \rho l(t) \text { for } t \in[0,1]
$$


We consider the boundary value problem

$$
\begin{gathered}
-u^{\prime \prime}=g(t, u \wedge \chi)+\mu h(t, u \wedge X), \\
u(0)=u(1)=0
\end{gathered}
$$

where $\mu \in(0, \lambda)$. Let $\tilde{g}_{1}(t, u)=g_{1}(t, u \wedge \chi), \tilde{g}_{2}(t, u)=g_{2}(t, u \wedge \chi), \tilde{h}_{1}(t, u)=h_{1}(t, u \wedge \chi)$, and $\tilde{h}_{2}(t, u)=h_{2}(t, u \wedge \chi)$. We easily prove that the conditions of [6, Theorem 1.2] are satisfied so (2.149) has a positive solution $u \in C^{1}(0,1) \cap C[0,1]$. We next prove that

$$
u(t) \leq X(t) \quad \text { for } t \in[0,1]
$$

Suppose (2.150) is not true. Let $y(t)=u(t)-\chi(t)$ and $\sigma \in(0,1)$ be the point where $y(t)$ attains its maximum over $(0,1)$. We have

$$
y(\sigma)>0, \quad y^{\prime \prime}(\sigma) \leq 0 .
$$

On the other hand, since $u(\sigma)>\chi(\sigma)$, we have

$$
\begin{aligned}
y^{\prime \prime}(\sigma) & =u^{\prime \prime}(\sigma)-\chi^{\prime \prime}(\sigma) \\
& =-g(\sigma, u \wedge \chi)-\mu h(\sigma, u \wedge \chi)+g(\sigma, \chi)+\lambda h(\sigma, \chi) \\
& =-g(\sigma, \chi(\sigma))-\mu h(\sigma, \chi(\sigma))+g(\sigma, \chi(\sigma))+\lambda h(\sigma, \chi(\sigma)) \\
& =(\lambda-\mu) h(\sigma, \chi(\sigma)) \\
& >0 .
\end{aligned}
$$

This is a contradiction, so

$$
u(t) \leq X(t) \quad \text { for } t \in[0,1]
$$

Thus we have

$$
\begin{gathered}
-u^{\prime \prime}=g(t, u)+\mu h(t, u), \\
u(0)=u(1)=0 .
\end{gathered}
$$

Let $\lambda_{3}^{*}=\sup \Lambda>0$. Then

(i) if $0<\lambda<\lambda_{3}^{*}$, (1.4) has at least one solution $u \in C[0,1] \cap C^{1}(0,1)$ and $u>0$ for $t \in(0,1)$;

(ii) if $\lambda>\lambda_{3}^{*}$, (1.4) has no solutions. 


\section{Example}

Example 3.1. Consider the boundary value problem

$$
\begin{gathered}
-u^{\prime \prime}=-\frac{1}{\sqrt{u}}+\lambda q(u) \quad \forall 0<t<1, \\
u(0)=u(1)=0,
\end{gathered}
$$

where $\lambda>1$.

Define $\left\{x_{n}\right\}_{n=1}^{\infty}$ as $x_{1}=2, x_{2 n}=x_{2 n-1}^{4}, x_{2 n+1}=x_{2 n}+1$, and

$$
q(r)= \begin{cases}r^{2}, & \text { if } r \in[0,2], \\ x_{2 n-1}^{2}, & \text { if } r \in\left[x_{2 n-1}, x_{2 n}\right], \\ \frac{x_{2 n+1}^{2}-\sqrt{x_{2 n}}}{x_{2 n+1}-x_{2 n}}\left(r-x_{2 n}\right)+\sqrt{x_{2 n},} & \text { if } r \in\left[x_{2 n}, x_{2 n+1}\right] .\end{cases}
$$

Then, Theorem 1.1 implies that there exists $\lambda_{1}^{*}>0$ such that for every $\lambda \geq \lambda_{1}^{*}$, (3.1) has at least one positive solution $u \in C[0,1] \cap C^{1}(0,1)$ and $u>0$ for $t \in(0,1)$.

To see this, let

$$
\begin{gathered}
g_{1}(t, r)=g_{2}(t, r)=\frac{1}{\sqrt{r}} \quad \text { for }(t, r) \in(0,1) \times(0, \infty), \\
h_{2}(t, r)=q(r) \text { for }(t, r) \in(0,1) \times(0, \infty), \\
h_{1}(t, r)= \begin{cases}\sqrt{r} & \text { for }(t, r) \in(0,1) \times(16, \infty), \\
q(r) & \text { for }(t, r) \in(0,1) \times(0,16) .\end{cases}
\end{gathered}
$$

It is easy to see that $(G 1),(H 1),(H 2)$, and $(H 3)$ are satisfied.

For all $r_{2}>r_{1}>0$, let $\gamma(t)=1 / 2 r_{1} \sqrt{r_{1}}$. Then $g_{2}(t, r)+\left(1 / 2 r_{1} \sqrt{r_{1}}\right) r$ is increasing in $\left(r_{1}, r_{2}\right)$.

On the other hand, $a_{1}=1+\int_{0}^{1}(1 / \sqrt{s}) d s=3$ and let $R_{j}=x_{2 j}-3$, so we have

$$
\begin{aligned}
\lim _{j \rightarrow \infty} \frac{h_{2}\left(s, R_{j}+a_{1}\right)}{R_{j}} & =\lim _{j \rightarrow \infty} \frac{h_{2}\left(s, x_{2 j}\right)}{x_{2 j}} \cdot \frac{x_{2 j}}{x_{2 j}-3} \\
& =\lim _{j \rightarrow \infty} \frac{\sqrt{x_{2 j}}}{x_{2 j}} \cdot \frac{x_{2 j}}{x_{2 j}-3} \\
& =0 .
\end{aligned}
$$

Thus (G2) and (H4) are satisfied. Then Theorem 1.1 implies that there exists $\lambda_{1}^{*}>0$ such that for every $\lambda \geq \lambda_{1}^{*}$, (3.1) has at least one positive solution $u \in C[0,1] \cap C^{1}(0,1)$ and $u>0$ for $t \in(0,1)$. 
Example 3.2. Consider the boundary value problem

$$
\begin{gathered}
-u^{\prime \prime}=g(t, u)+\lambda h(t, u), \quad t \in(0,1), \\
u(0)=0=u(1),
\end{gathered}
$$

where

$$
\begin{gathered}
g(t, r)=\left\{\begin{array}{cl}
\frac{1}{r^{\alpha}}\left|\sin \frac{1}{r}\right|, & 0<r \leq \frac{1}{\pi} \\
-\frac{1}{r^{\alpha}} \sin \frac{1}{r}, & \frac{1}{\pi}<r
\end{array}\right. \\
h(t, r)=r^{2},
\end{gathered}
$$

with $\alpha>0$. Then Theorem 1.2 guarantees that there exists $\lambda_{2}^{*}>0$ such that

(i) if $0<\lambda<\lambda_{2}^{*}$, (3.5) has at least one solution $u \in C[0,1] \cap C^{1}(0,1)$ and $u>0$ for $t \in(0,1)$;

(ii) if $\lambda>\lambda_{2}^{*}$, (3.5) has no solutions.

To see this, let $\beta=\min \{1 / 2, \alpha / 2\}, g_{1}(t, r)=1 / r^{\beta}+\pi^{\alpha}$, and $g_{2}(t, r)=1 / r^{\alpha}$, for $(t, r) \in$ $(0,1) \times(0, \infty)$, and $h_{1}(t, r)=h_{2}(t, r)=r^{2}$, for $(t, r) \in(0,1) \times[0, \infty)$. Notice that $(G 1),(H 1)$, and $(H 2)$ are satisfied.

For all $r_{2}>r_{1}>0$, let

$$
r(t) \equiv \sup _{r \in \Lambda}\left|\frac{\partial g}{\partial r}\right|+1<\infty
$$

where $\Lambda=\left(r_{1}, r_{2}\right) \backslash\{n \pi \mid n \in N\}$, so we have $g(t, r)+\gamma(t) r$ is increasing in $\left(r_{1}, r_{2}\right)$.

Let $c_{1}=1 / \pi$ and we have

$$
0 \leq g(t, r), \quad t \in(0,1), 0<r<c_{1} .
$$

Let $n_{0}$ be fixed such that

$$
2^{1 /(\alpha-\beta)}<n_{0} \pi+\frac{\pi}{6}
$$

Let $c_{2} \in\left(0, c_{1}\right)$ be such that

$$
c_{2}^{3}<\frac{1}{2(2-\beta) \pi^{1-\beta}} \sum_{n=n_{0}}^{\infty}\left[\left(\frac{6}{6 n+1}\right)^{2-\beta}-\left(\frac{6}{6 n+5}\right)^{2-\beta}\right],
$$


and we have for $n \geq n_{0}, r \in\left(0, c_{2}\right)$,

$$
\frac{1}{(r t)^{\alpha}}\left|\sin \frac{1}{r t}\right| \geq \frac{1}{(r t)^{\beta}} \quad \text { for } t \in\left[\frac{1}{r(n \pi+5 \pi / 6)}, \frac{1}{r(n \pi+\pi / 6)}\right] \text {. }
$$

Also we have

$$
\begin{aligned}
\int_{0}^{1} t(1-t) \bar{g}_{m}(t, r l(t)) d t & \geq \int_{0}^{1 / 2} t(1-t) \bar{g}_{m}(t, r l(t)) d t \\
& \geq \frac{1}{2} \int_{0}^{1 / 2} t \bar{g}_{m}(t, r l(t)) d t \\
& \geq \frac{1}{2} \sum_{n=n_{0}}^{\infty} \int_{1 / r(n \pi+5 \pi / 6)}^{1 / r(n \pi+\pi / 6)} t \frac{1}{(r t)^{\beta}} d t \\
& \geq \frac{1}{2 r^{\beta}} \sum_{n=n_{0}}^{\infty} \int_{1 / r(n \pi+5 \pi / 6)}^{1 / r(n \pi+\pi / 6)} t^{1-\beta} d t \\
& =\frac{1}{2 r^{2}(2-\beta) \pi^{2-\beta}} \sum_{n=n_{0}}^{\infty}\left[\left(\frac{6}{6 n+1}\right)^{2-\beta}-\left(\frac{6}{6 n+5}\right)^{2-\beta}\right] \\
& \geq r \pi .
\end{aligned}
$$

Thus (G5) is satisfied.

\section{Acknowledgment}

This research is supported by NNSF of China (10871059).

\section{References}

[1] R. P. Agarwal and D. O'Regan, Singular Differential and Integral Equations with Applications, Kluwer Academic Publishers, Dordrecht, The Netherlands, 2003.

[2] R. P. Agarwal and D. O'Regan, "Twin solutions to singular Dirichlet problems," Journal of Mathematical Analysis and Applications, vol. 240, no. 2, pp. 433-445, 1999.

[3] D. O'Regan, Theory of Singular Boundary Value Problems, World Scientific, River Edge, NJ, USA, 1994.

[4] P. Habets and F. Zanolin, "Upper and lower solutions for a generalized Emden-Fowler equation," Journal of Mathematical Analysis and Applications, vol. 181, no. 3, pp. 684-700, 1994.

[5] H. Lü, D. O’Regan, and R. P. Agarwal, "An approximation approach to eigenvalue intervals for singular boundary value problems with sign changing nonlinearities," Mathematical Inequalities and Applications, vol. 11, no. 1, pp. 81-98, 2007.

[6] H. Lü, D. O’Regan, and R. P. Agarwal, "Existence to singular boundary value problems with sign changing nonlinearities using an approximation method approach," Applications of Mathematics, vol. 52, no. 2, pp. 117-135, 2007. 